in vivo $34: 2913-2917$ (2020)

doi:10.21873/invivo.12120

\title{
The Influence of the Metabolic Syndrome on Early Postoperative Outcomes of Patients With Advanced-stage Endometrial Cancer
}

\author{
NICOLAE BACALBASA ${ }^{1,2,3}$, CAMELIA DIACONU $^{4,5}$, LAURA ILIESCU $^{4,6}$, CORNEL SAVU $^{7,8}$, CARMEN SAVU $^{9}$, \\ CRISTIAN BALALAU ${ }^{10,11}$, MIHAI DIMITRIU ${ }^{1,12}$, ALEXANDRU FILIPESCU ${ }^{1,13}$, OVIDIU GABRIEL BRATU ${ }^{14,15}$, \\ ADRIAN NEACSU ${ }^{16}$, DRAGOS CRETOIU ${ }^{1,17}$, IOANA HALMACIU ${ }^{18}$ and IRINA BALESCU ${ }^{19}$ \\ ${ }^{1}$ Department of Obstetrics and Gynecology, \\ Carol Davila University of Medicine and Pharmacy, Bucharest, Romania; \\ ${ }^{2}$ Department of Obstetrics and Gynecology, I. Cantacuzino Clinical Hospital, Bucharest, Romania; \\ ${ }^{3}$ Department of Visceral Surgery, Center of Excellence in Translational \\ Medicine Fundeni Clinical Institute, Bucharest, Romania; \\ ${ }^{4}$ Department of Internal Medicine, Carol Davila University of Medicine and Pharmacy, Bucharest, Romania; \\ ${ }^{5}$ Department of Internal Medicine, University Emergency Hospital Bucharest, Bucharest, Romania; \\ ${ }^{6}$ Department of Internal Medicine, Fundeni Clinical Institute, Bucharest, Romania; \\ ${ }^{7}$ Department of Thoracic Surgery, Carol Davila University of Medicine and Pharmacy, Bucharest, Romania; \\ ${ }^{8}$ Department of Thoracic Surgery, Marius Nasta Institute of Pneumonology Bucharest, Romania; \\ ${ }^{9}$ Department of Anesthesiology, Fundeni Clinical Institute, Bucharest, Romania; \\ ${ }^{10}$ Department of Surgery, Pantelimon Clinical Hospital, Bucharest, Romania; \\ ${ }^{11}$ Department of Surgery, Carol Davila University of Medicine and Pharmacy, Bucharest, Romania; \\ ${ }^{12}$ Department of Obstetrics and Gynecology, St. Pantelimon Emergency Clinical Hospital, Bucharest, Romania; \\ ${ }^{13}$ Department of Obstetrics and Gynecology, Elias Emergency Hospital, Bucharest, Romania; \\ ${ }^{14}$ Department of Urology, Carol Davila University of Medicine and Pharmacy, Bucharest, Romania; \\ ${ }^{15}$ Department of Urology, Emergency Central Military Hospital, \\ Academy of Romanian Scientists, Bucharest, Romania; \\ ${ }^{16}$ Department of Obstetrics and Gynecology, St. John Emergency Clinical Hospital, Bucharest, Romania; \\ ${ }^{17}$ Alessandrescu-Rusescu National Institute of Mother and Child Health, \\ Fetal Medicine Excellence Research Center, Bucharest, Romania; \\ ${ }^{18}$ Department of Anatomy, George Emil Palade University of Medicine, \\ Pharmacy, Science and Technology, Târgu Mureș, Romania; \\ ${ }^{19}$ Department of Surgery, Ponderas Academic Hospital, Bucharest, Romania
}

\begin{abstract}
Background/Aim: Endometrial cancer is one of the most commonly encountered malignancies among obese women worldwide, a strong causality relationship being established between the two entities. Furthermore, obesity is also associated with metabolic syndrome; the aim of this
\end{abstract}

This article is freely accessible online.

Correspondence to: Irina Balescu, Ponderas Academic Hospital, Bucharest, Nicolae Caramfil 85a Street, Romania. Tel: +40 724077709, e-mail: irina.balescu@ponderas-ah.ro

Key Words: Endometrial cancer, metabolic syndrome, obesity. study was to investigate the effect of metabolic syndrome on the postoperative outcomes of patients with endometrial cancer. Patients and Methods: Data of 23 patients diagnosed with endometrial cancer and metabolic syndrome were retrospectively reviewed and compared to the those of a control group of patients diagnosed with endometrial cancer in the absence of metabolic syndrome. Results: Patients in the first group presented significantly higher values of body mass index when compared to the control group. There were no significant differences in terms of stage, histopathological subtype or degree of differentiation between the two groups. The completeness of cytoreduction was lower among patients with metabolic syndrome, however, this did not reach statistical significance $(p=0.08)$. Although the rate of 
complete debulking was lower among those with metabolic syndrome, the rates of postoperative complications were significantly higher. Conclusion: The association of metabolic syndrome significantly influences the risk of postoperative complications in patients with endometrial cancer; moreover, in certain cases, it might preclude the achievement of freedom from residual disease.

An association between obesity and metabolic syndrome has been widely demonstrated: patients with higher body mass indices bear a higher risk of developing severe metabolic conditions such as diabetes mellitus, atherosclerosis, dyslipidemia, sleep apnea and chronic inflammation. All these comorbidities can further induce the development of chronic inflammatory status, which will influence the risk of developing further malignancies, endometrial cancer being the most common one in females (1-3). A positive association between obesity, metabolic syndrome and premalignant and even malignant gynecological conditions has been widely demonstrated. Moreover, it seems that this association significantly influences the perioperative and postoperative outcomes of patients submitted to surgery for endometrial cancer (4-6). The aim of this study was to analyze the influence of obesity and metabolic syndrome on the early postoperative outcomes of patients submitted to surgery for advanced-stage endometrial cancer.

\section{Patients and Methods}

After obtaining the approval of the Ethical Committee (no. $53 / 2020$ ), data of patients submitted to surgery for advanced-stage endometrial cancer were retrospectively reviewed. Between 2019 and 2020, 23 patients with metabolic syndrome and endometrial cancer were submitted to surgery. Their outcomes were compared to those of a similar group of 23 consecutive normal-weight patients diagnosed with similar stages of endometrial cancer and in whom metabolic syndrome was not associated. The preoperative classification of the stage of disease was performed in all cases according to the International Federation of Obstetrics and Gynecology (FIGO) classification (7), while postoperative complications were classified according to the Clavien-Dindo scale (8). In order to define metabolic syndrome, the statement of the International Diabetes Federation Task Force on epidemiology and Prevention 2009 was used (9). According to this definition, clinical diagnosis of metabolic syndrome should include the following criteria: Elevated waist circumference, higher amounts of circulating triglycerides or administration of drugs for lowering the serum levels of triglycerides, reduced serum levels of high-density lipoproteins (or administration of drugs for increasing these levels), elevated blood pressure (or a history of antihypertensive treatment), and elevated serum glucose (or antidiabetic treatment) (9). Patients were considered as obese when they had a body mass index (BMI) higher than $30 \mathrm{~kg} / \mathrm{m}^{2}$ (10). In all cases, the intent was to obtain a state of no residual disease (defined as R0 resection); cases in which the residual volume was between 0.1 and $1 \mathrm{~cm}$ were considered to have been submitted to R1 resection, while in cases in which the residual volume was larger than $1 \mathrm{~cm}$ were defined as having had an $\mathrm{R} 2$ resection.

The endpoints of the study consisted of determining if a significant correlation could be established between the presence of metabolic syndrome, the type of cytoreduction and any early postoperative complications. In order to study the presence of these correlation, univariate analysis was performed in which $p$-values lower than 0.05 . were considered as having statistical significance.

\section{Results}

There were no significant differences in terms of age between the two groups, the median age being 54 (range $=51-71$ years) years among patients with metabolic syndrome and 51 years (range $=43-68$ years) among patients with no other comorbidities. The stage at initial diagnosis was similar for the two groups, 15 cases in the first group and 17 in the second group being diagnosed with FIGO stage IIIC endometrial cancer, while the remaining eight and six cases, respectively, were diagnosed with FIGO stage IV of disease. BMI was significantly ( $p=0.02)$ higher among patients in the first group $\left(38.5 \mathrm{~kg} / \mathrm{m}^{2}\right)$ compared with the second $\left(23 \mathrm{~kg} / \mathrm{m}^{2}\right)$. In all cases, surgery with curative intent was attempted; however, this was only achieved in 18 cases among the first group and 22 cases in the second. Histopathological studies demonstrated that patients in the group with metabolic syndrome were significantly more frequently diagnosed with type I endometrial cancer (this histopathological subtype being encountered in 21 versus eight cases without metabolic syndrome, $p=0.043$ ). Regarding the degree of differentiation, no significant difference was found between the two groups. Intraoperative details are shown in Table I.

As observed from the Table I, there was no significant difference in terms of length of surgery and intraoperative blood loss between the two groups; this aspect is rather explained by the fact that patients with metabolic syndrome and increased BMI were more likely to be submitted to less extensive procedures in order to try to diminish the risk of perioperative complications. This aspect was also responsible for the fact that the radicality of cytoreduction was lower among patients with metabolic syndrome; however, this did not reach any statistical significance.

Patients with metabolic syndrome experienced a significantly higher rate of postoperative complications as well as a greater length of hospital stay. However, the necessity for reoperation was similar for the two groups, while the 30-day mortality was nil. Postoperative details are presented in Table II.

The most commonly encountered complications were represented by Clavien-Dindo grade I complications and were wound infections and urinary tract infections, which were significantly more commonly encountered in patients with metabolic syndrome. The proportion of patients requiring postoperative reoperation (defined as those with IIIb 
Table I. Intraoperative characteristics of patients submitted to surgery for advanced-stage endometrial cancer.

\begin{tabular}{|c|c|c|c|}
\hline \multirow[b]{2}{*}{ Characteristic } & \multicolumn{2}{|c|}{ Patient group } & \multirow[b]{2}{*}{$p$-Value } \\
\hline & $\begin{array}{l}\text { Metabolic } \\
\text { syndrome }\end{array}$ & Control & \\
\hline \multicolumn{4}{|l|}{ Age, years } \\
\hline Median (range) & $54(51-71)$ & $51(43-68)$ & 0.07 \\
\hline \multicolumn{4}{|l|}{$\mathrm{BMI}, \mathrm{kg} / \mathrm{m}^{2}$} \\
\hline Median (range) & $38.5(24-46.3)$ & $23(17.3-33.4)$ & 0.02 \\
\hline \multicolumn{4}{|l|}{ FIGO stage at diagnosis, $\mathrm{n}$} \\
\hline IIIC & 15 & 17 & 0.43 \\
\hline IV & 8 & 6 & \\
\hline \multicolumn{4}{|l|}{ Histopathological type, $\mathrm{n}$} \\
\hline Type I & 21 & 8 & 0.043 \\
\hline Type II & 2 & 15 & \\
\hline \multicolumn{4}{|l|}{ Degree of differentiation, $\mathrm{n}$} \\
\hline Well-differentiated & 5 & 3 & 0.78 \\
\hline Moderately differentiated & 8 & 9 & \\
\hline Poorly differentiated & 10 & 11 & \\
\hline \multicolumn{4}{|l|}{ Type of resection, $n$} \\
\hline R0 & 18 & 22 & 0.08 \\
\hline $\mathrm{R} 1$ & 4 & 1 & \\
\hline $\mathrm{R} 2$ & 1 & 0 & \\
\hline \multicolumn{4}{|l|}{ Duration of surgery, min } \\
\hline Median (range) & $110(90-140)$ & $120(90-180)$ & 0.45 \\
\hline \multicolumn{4}{|l|}{ Estimated blood loss, ml } \\
\hline Median (range) & $350(200-400)$ & $310(150-400)$ & 0.63 \\
\hline
\end{tabular}

FIGO: International Federation of Obstetrics and Gynecology; BMI: body mass index. Bold values indicate statistical significance.

complications) were similar for the two groups; reoperation was needed in one case in each group and was caused by postoperative hemoperitoneum in both cases. Severe complications (defined as Clavien-Dindo grade IV complications) were encountered in a single case among patients of the metabolic group and consisted of severe pulmonary dysfunction in a morbidly obese $\left(\mathrm{BMI}=46.3 \mathrm{~kg} / \mathrm{m}^{2}\right)$ patient with chronic heart failure, pulmonary dysfunction and sleep apnea. The patient required intubation on the third postoperative day; however, her evolution was slightly favorable, extubation being possible on the $11^{\text {th }}$ postoperative day. The patient was discharged on the $16^{\text {th }}$ postoperative day.

\section{Discussion}

Introduced for the first time by Reaven in 1988 (11), the term 'metabolic syndrome' (also known as insulin resistance syndrome or X syndrome) represents an association between cardiovascular risk factors, diabetes and obesity which has been redefined several times in recent decades (11-14). Currently, the most recent definition of this syndrome was provided in 2009 by the International Diabetes Federation Task Force on Epidemiology and Prevention and consists of
Table II. Postoperative details of patients submitted to surgery for advanced-stage endometrial cancer.

\begin{tabular}{lccc}
\hline & \multicolumn{2}{c}{ Patient group } & \\
\cline { 2 - 3 } Characteristic & $\begin{array}{l}\text { Metabolic } \\
\text { syndrome }\end{array}$ & Control & $p$-Value \\
& & & \\
\hline Clavien-Dindo complications & $13 \%$ & $4 \%$ & $\mathbf{0 . 0 2 3}$ \\
Grade Ic & $8 \%$ & $4 \%$ & $\mathbf{0 . 0 4}$ \\
Grade II & $8 \%$ & $4 \%$ & 0.04 \\
Grade IIIa & $4 \%$ & $4 \%$ & $>0.99$ \\
Grade IIIb & $4 \%$ & $0 \%$ & 0.0001 \\
Grade IVa & $0 \%$ & $0 \%$ & - \\
Grade IVb & & & \\
Hospital stay, days & $7(5-16)$ & $5(4-7)$ & 0.03 \\
Median (range) & & & \\
\hline
\end{tabular}

Bold values indicate statistical significance.

the association of any of the three following factors: Waist circumference higher than $80 \mathrm{~cm}$ for Asian populations and $88 \mathrm{~cm}$ for European populations, triglyceride level higher than $150 \mathrm{mg} / \mathrm{dl}$, high-density lipoprotein cholesterol level lower than $50 \mathrm{mg} / \mathrm{dl}$, systolic blood pressure higher than 130 $\mathrm{mmHg}$ /diastolic blood pressure higher than $85 \mathrm{mmHg}$, and fasting plasma glucose level higher than $100 \mathrm{mg} / \mathrm{dl}$ (9). Therefore it is widely accepted that metabolic syndrome represents a significant risk factor for further development of severe cardiovascular, respiratory and diabetes-related complications; moreover, it has been demonstrated that presence of metabolic syndrome represents a significant risk factor for the development of certain neoplastic diseases such as colorectal, breast and endometrial cancer (10-13).

An interesting study conducted on this issue by Rosato et al. demonstrated that patients with metabolic syndrome have an odds ratio ranging between 1.67 and 2.77 of developing endometrial cancer when compared to patients with no such comorbidity (15). This aspect is explained through the fact that patients with metabolic syndrome usually present higher circulating levels of estrogens, insulin and fatty acids. An increased level of circulating insulin is also associated with an increased amount of insulin-like growth factor-1, which is widely recognized as a significant promoter of cell proliferation and malignant transformation. Moreover, the presence of high amounts of these substances will lead to the development of high levels of proinflammatory molecules, increasing in this way the risk of malignant transformation of certain tissues such as the endometrium (16-19).

However, it seems that metabolic syndrome not only induces the development of endometrial cancer but also influences the short- and long-term outcomes of these patients. In the study conducted by Mahdi et al. on 4,000 patients diagnosed with endometrial cancer, the influence of 
the age and comorbidities on the 30 day morbidity and mortality risk was investigated (20). According to their study, the perioperative risks were not influenced by patient age, no significant differences being observed between different age groups of patients (lower than 60 years, 60-69 years, 7079 years and older than 80 years); in this respect, the authors recommend surgery should not be routinely omitted for elderly patients, age alone not being a significant risk factor for postoperative complications. However, they pointed out that the presence of other significant comorbidities, most of them being part of metabolic syndrome (cardiac comorbidities, respiratory comorbidities, and diabetes mellitus), was significantly associated with poorer postoperative outcomes. These risk factors seemed to be significantly associated with increased age; therefore, the authors concluded that not age itself but the association of these types of comorbidities should be carefully analyzed in patients with endometrial cancer submitted to surgery (20).

Another important issue in postoperative complications in endometrial cancer is related to the frequent association of obesity. In a study on 627 patients diagnosed with endometrial cancer, Bowman et al. demonstrated that obesity was significantly associated with higher rates of complications, such as wound infection, and a higher necessity for antibiotic administration; other complications which were more frequently encountered in obese patients were cardiac, renal and pulmonary complications, a previous history of metabolic syndrome also being a possible cause for the development of such events (21).

An interesting study conducted on the issue of the correlation between metabolic syndrome and postoperative outcomes of patients with endometrial cancer was published by Nagle et al. in 2018 (22). The study included 1,359 patients diagnosed with endometrial cancer; after a median follow-up of 7.1 years, 179 patients had died, 123 of them due to endometrial cancer. The association of diabetes and obesity significantly influenced overall survival, all-cause mortality being higher among this group, while diabetic patients had a significantly higher rate of cancer-related death. Moreover, the association of more than two comorbidities (after excluding diabetes) was also associated with an increased rate of cancerrelated mortality. Interestingly, the association between diabetes mellitus and aggressive biological subtypes of tumors (highgrade endometrial carcinoma, non-endometroid endometrial carcinoma) led to a significantly higher rate of cancer-related death when compared to the other causes of death (22).

\section{Conclusion}

The association between obesity, endometrial cancer and metabolic syndrome has been widely demonstrated so far, multiple endocrine and inflammatory mechanisms being responsible for this correlation. The presence of preoperative complications such as obesity, cardiac, pulmonary diseases and diabetes mellitus (widely known as metabolic syndrome) seems to significantly influence the early postoperative outcomes of patients with endometrial cancer. Moreover, these metabolic and functional disorders also seem to influence the long-term outcomes of patients with endometrial cancer.

\section{Conflicts of Interest}

The Authors declare that there are no conflicts of interest regarding this study.

\section{Authors' Contributions}

NB: Performed the surgical procedures; IB: prepared the article; $C D$, LI, CB, DC, IH: performed data analysis; OGB, AF, Cornel S, Carmen $\mathrm{S}, \mathrm{MD}, \mathrm{AN}$ : were part of the surgical team; DC: advised about the oncological outcome; $\mathrm{NB}, \mathrm{CB}$ : revised the final draft of the article.

\section{Acknowledgements}

This work was supported by the project entitled "Multidisciplinary Consortium for Supporting the Research Skills in Diagnosing, Treating and Identifying Predictive Factors of Malignant Gynecologic Disorders", project number PN-III-P1-1.2-PCCDI2017-0833.

\section{References}

1 Obesity: preventing and managing the global epidemic. Report of a WHO consultation. World Health Organ Tech Rep Ser 894 i-253, 2000. PMID: 11234459.

2 Clinical Guidelines on the Identification, Evaluation, and Treatment of Overweight and Obesity in Adults-The Evidence Report. National Institutes of Health. Obes Res 6 Suppl 2: 51S209S, 1998. PMID: 9813653.

3 Tomescu DR, Popescu M, Dima SO, Bacalbasa N and BubenekTurconi S: Obesity is associated with decreased lung compliance and hypercapnia during robotic assisted surgery. J Clin Monit Comput 31(1): 85-92, 2017. PMID: 26823286. DOI: 10.1007/ s10877-016-9831-y

4 Balalau DO, Sima RM, Bacalbasa N, Ples L and Stanescu AD: Emergency peripartum hysterectomy, physical and mental consequences: A 6-year study. J Mind Med Sci 3(1): 8, 2016.

5 Balalau DO, Sima RM, Bacalbasa N, Banu P, Balalau C, Ples L and Stanescu AD: High-grade cervical dysplasia in pregnancy psychological and medical challenges. J Mind Med Sci 4(1): 6, 2017. DOI: $10.22543 / 7674.41 . P 2430$

6 Bacalbasa N, Balescu I, Dragan I, Banceanu G, Suciu I and Suciu N: Endometrial adenocarcinoma presenting as hematometra with underlying thickened endometrial lining in a postmenopausal woman - a case report. Anticancer Res 36(5): 2353-2357, 2016. PMID: 27127143.

7 Creasman W: Revised FIGO staging for carcinoma of the endometrium. Int J Gynaecol Obstet 105(2): 109, 2009. PMID: 19345353. DOI: $10.1016 /$ j.ijgo.2009.02.010

8 Clavien PA, Barkun J, de Oliveira ML, Vauthey JN, Dindo D, Schulick RD, de Santibanes E, Pekolj J, Slankamenac K, Bassi C, Graf R, Vonlanthen R, Padbury R, Cameron JL and Makuuchi 
M: The Clavien-Dindo classification of surgical complications: Five-year experience. Ann Surg 250 (2): 187-196, 2009. PMID: 19638912. DOI: 10.1097/SLA.0b013e3181b13ca2

9 Alberti KG, Eckel RH, Grundy SM, Zimmet PZ, Cleeman JI, Donato KA, Fruchart JC, James WP, Loria CM and Smith SC, Jr.: Harmonizing the metabolic syndrome: A joint interim statement of the International Diabetes Federation Task Force on Epidemiology and Prevention; National Heart, Lung, and Blood Institute; American Heart Association; World Heart Federation; International Atherosclerosis Society; and International Association for the Study of Obesity. Circulation 120(16): 1640-1645, 2009. PMID: 19805654. DOI: 10.1161/CIRCULATIONAHA.109.192644

10 WHO Global Health Observatory (GHO) data - Mean Body Mass Index (BMI). World Health Organization. Retrieved 5 February 2019. Available at: https://www.who.int/gho/ncd/ risk_factors/bmi_text/en/ [Last accessed on May, 21st 2020]

11 Reaven GM: Banting lecture 1988. Role of insulin resistance in human disease. Diabetes 37(12): 1595-1607, 1988. PMID: 3056758. DOI: $10.2337 /$ diab.37.12.1595

12 Third Report of the National Cholesterol Education Program (NCEP) Expert Panel on Detection, Evaluation, and Treatment of High Blood Cholesterol in Adults (Adult Treatment Panel III) Final Report. Circulation 106(25): 3143-3421, 2002. PMID: 12485966.

13 Grundy SM, Cleeman JI, Daniels SR, Donato KA, Eckel RH, Franklin BA, Gordon DJ, Krauss RM, Savage PJ, Smith SC, Jr., Spertus JA and Costa F: Diagnosis and management of the metabolic syndrome: An American Heart Association/National Heart, Lung, and Blood Institute Scientific Statement. Circulation 112(17): 2735-2752, 2005. PMID: 16157765. DOI: 10.1161/CIRCULATIONAHA.105.169404

14 Zimmet P, Magliano D, Matsuzawa Y, Alberti G and Shaw J: The metabolic syndrome: A global public health problem and a new definition. J Atheroscler Thromb 12(6): 295-300, 2005. PMID: 16394610. DOI: 10.5551/jat.12.295

15 Rosato V, Zucchetto A, Bosetti C, Dal Maso L, Montella M, Pelucchi C, Negri E, Franceschi S and La Vecchia C: Metabolic syndrome and endometrial cancer risk. Ann Oncol 22(4): 884889, 2011. PMID: 20937645. DOI: 10.1093/annonc/mdq464
16 Cook L, Weiss NS, Doherty JA and Chen C: Endometrial cancer. In: Cancer Epidemiology and Prevention, Third Edition. Schottenfeld D and Fraumeni JF (eds). New York: Oxford University Press, pp. 1027-1043, 2006.

17 Eckel RH, Grundy SM and Zimmet PZ: The metabolic syndrome. Lancet 365(9468): 1415-1428, 2005. PMID: 15836891. DOI: $10.1016 / \mathrm{S} 0140-6736(05) 66378-7$

18 Murphy LJ: Growth factors and steroid hormone action in endometrial cancer. J Steroid Biochem Mol Biol 48(5-6): 419-423, 1994. PMID: 8180102. DOI: 10.1016/0960-0760(94)90189-9

19 Thiet MP, Osathanondh R and Yeh J: Localization and timing of appearance of insulin, insulin-like growth factor-I, and their receptors in the human fetal mullerian tract. Am J Obstet Gynecol 170(1 Pt 1): 152-156, 1994. PMID: 8296817. DOI: 10.1016/s0002-9378(94)70401-5

20 Mahdi H, Lockhart D and Maurer KA: Impact of age on 30-day mortality and morbidity in patients undergoing surgery for endometrial cancer. Gynecol Oncol 137(1): 106-111, 2015. PMID: 25640765. DOI: 10.1016/j.ygyno.2015.01.543

21 Bouwman F, Smits A, Lopes A, Das N, Pollard A, Massuger L, Bekkers R and Galaal K: The impact of BMI on surgical complications and outcomes in endometrial cancer surgery-an institutional study and systematic review of the literature. Gynecol Oncol 139(2): 369-376, 2015. PMID: 26407479. DOI: 10.1016/j.ygyno.2015.09.020

22 Nagle CM, Crosbie EJ, Brand A, Obermair A, Oehler MK, Quinn M, Leung Y, Spurdle AB and Webb PM: The association between diabetes, comorbidities, body mass index and all-cause and cause-specific mortality among women with endometrial cancer. Gynecol Oncol 150(1): 99-105, 2018. PMID: 29706522. DOI: 10.1016/j.ygyno.2018.04.006
Received April 29, 2020

Revised May 21, 2020

Accepted May 29, 2020 